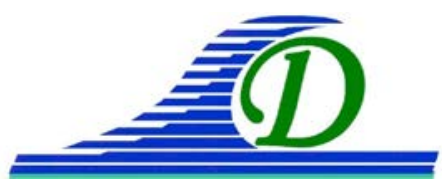
XIII ${ }^{\text {èmes }}$ Journées Nationales Génie Côtier - Génie Civil Dunkerque, 2-4 juillet 2014

DOI:10.5150/jngcgc.2014.019 (c) Editions Paralia CFL

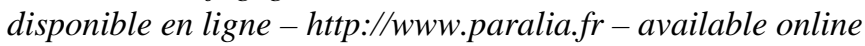

\title{
Modélisation numérique du niveau instantané de la mer à la côte au cours de phénomènes paroxysmaux (Wan-Tzu-Liao, Taïwan)
}

\author{
Fabien RETIF ${ }^{1}$, Frédéric BOUCHETTE ${ }^{1}$, Jiing-Yih LIOU ${ }^{2}$, Samuel MEULE $^{3}$, \\ Héloïse MICHAUD ${ }^{4}$, Li-Ching LIN $^{5}$, Kao-Shu HWANG ${ }^{2}$, Nans BUJAN ${ }^{2}$, \\ Hwung-Hweng HWUNG ${ }^{6}$, Patrick MARSALEIX ${ }^{7}$, Claude ESTOURNEL ${ }^{7}$, \\ Cyril NGUYEN $^{7}$, Caroline ULSES ${ }^{7}$
}

1. Géosciences-M, UMR 5243, University Montpellier 2 \& CNRS, 34095 Montpellier, France.fabien.retif@univ-montp2.fr

2. Tainan Hydraulics Laboratory, National Cheng Kung University, Taiwan.

3. Aix-Marseille Université, CNRS, IRD, CEREGE UM34, Aix en Provence, France.

4. SHOM, 42 avenue Gaspar Coriolis, 31157 Toulouse Cedex, France.

5. International Wave Dynamics Research Center, National Cheng Kung University, Taiwan.

6. Department of Hydraulic and Ocean Engineering, National Cheng Kung University, Taiwan.

7. Laboratoire d'Aérologie - CNRS \& University of Toulouse, 14, Avenue Edouard Belin, 31400 Toulouse, France.

\section{Résumé :}

Des simulations académiques de l'hydrodynamique 3D ont été réalisées sur une zone s'étendant à environ $600 \mathrm{~km}$ autour de l'île de Taïwan, pour la période de Septembre 2011 à Octobre 2011. Ces simulation permettent de discuter les mécanismes hydrodynamiques régionaux fondamentaux à l'origine de l'élévation du niveau d'eau le long de la barrière sableuse de Wan-Tzu-Liao.

Les simulations sont basées sur le modèle de courantologie 3D SYMPHONIE, intégrant convenablement la totalité des forçages météo-marins ayant un rôle sur la circulation et le niveau d'eau à la côte : vent, flux atmosphériques, circulation grande échelle, marée, pression atmosphérique, rivières et vagues. Ces simulations présentent l'originalité d'embarquer les développements les plus récents en matière de couplage 3D vent/ vague. Par ailleurs, dans le cadre du projet franco-taïwanais KUNSHEN, un ensemble d'appareils a été déployé le long d'un profil cross-shore garantissant un très bon contrôle de l'hydrodynamique sur la barrière sableuse de Wan-Tzu-Liao de Septembre 2011 à Décembre 2012. Sur ce profil, les mesures et les simulations de niveau d'eau, des courants de surface et de fond ont été comparées. Modulo l'effet des vagues, les simulations reproduisent correctement les mesures, avec une qualité équivalente à celle obtenue par MICHAUD et al. (2012) sur une plage du golfe du Lion (France).

Mots-clés : Niveau d'eau, Vent, vague, Courant, Typhons, Conditions extrêmes. 


\section{Introduction}

Le détroit de Taïwan, délimité par la Chine continentale à l'Est et l'île de Taïwan à l'Ouest, est un plan d'eau de $180 \mathrm{~km}$ de large, $350 \mathrm{~km}$ de long et présentant une faible bathymétrie moyenne, de l'ordre de $80 \mathrm{~m}$ (figure 1). Au nord, le détroit rejoint l'Est de la mer de Chine au niveau d'un large plateau continental s'étendant sur plusieurs centaines de km. Au Sud, le détroit débouche sur le Nord de la mer de Chine; le plateau continental se rétrécit progressivement du Nord au Sud, sur une centaine de $\mathrm{km}$. Le détroit constitue donc un lieu d'échange entre les eaux des mers de Chine de l'Est et de Chine du Sud (CHIOU et al., 2010).

L'île de Taïwan est située sur le passage ou dans la zone d'influence directe de la plupart des typhons provenant du Nord-Ouest du Pacifique, avec une moyenne annuelle de trois ou quatre typhons qui la frappent directement (LIN et al., 2009) et une vingtaine qui passent à proximité. Comme le reste de lîle, le Sud-Ouest de Taïwan dans la région de Tainan où se situe la barrière de Wan-Tzu-Liao est régulièrement exposé à ces événements paroxysmaux. La barrière sableuse de Wan-Tzu-Liao protège un système lagunaire à caractère micro-tidal. Elle présente une largeur entre 100 et 500 mètres et s'étant sur un peu plus de $8 \mathrm{~km}$ le long de la côte. Cette zone est fortement affectée par le passage des typhons et on observe des déplacements importants des barres d'avantcôte (CAMPMAS et al., 2014).

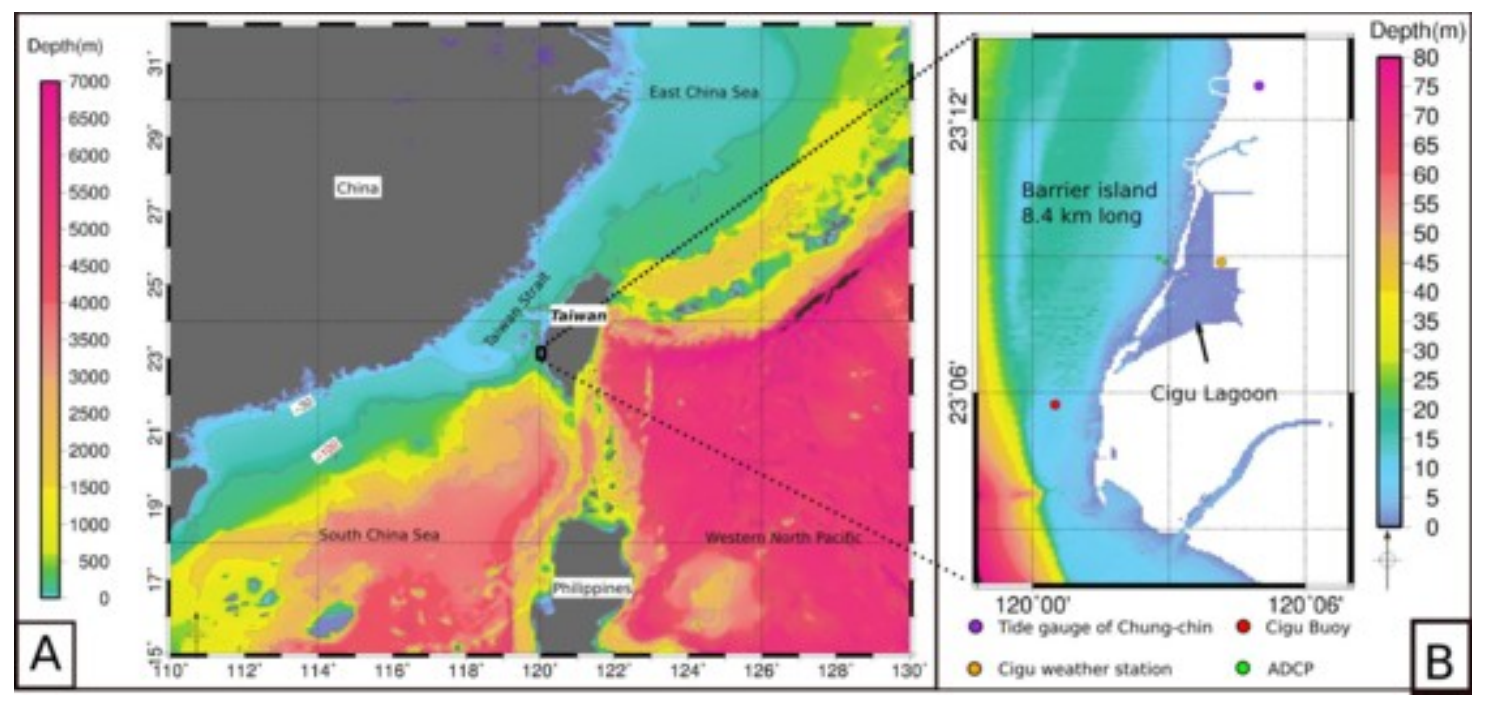

Figure 1. A) La bathymétrie du détroit de Taïwan ; B) La bathymétrie de la barrière sableuse de Wan-Tzu-Liao et la position des instruments utilisés pour la validation des simulations.

Notre objectif est de mieux caractériser la contribution des différents paramètres météomarins ayant un impact sur le niveau d'eau à la côte. Même si les mécanismes hydrodynamiques sont à l'heure actuelle bien connus en conditions normales, ils restent 


\section{XIII ${ }^{\text {èmes }}$ Journées Nationales Génie Côtier - Génie Civil \\ Dunkerque, 2-4 juillet 2014}

difficile à restituer convenablement dans les modélisations ; en effet, modéliser les extrêmes requiert des données de haute résolution spatiale et temporelle car ces processus sont par essence court et avec une grande variabilité en intensité. La mise en place des simulations est donc lourdes. Nous avons réalisé une simulation de l'hydrodynamique et des niveaux d'eau à la côte soumis à des conditions extrêmes en analysant les contributions de la marée, des courants globaux et des conditions atmosphériques à une échelle régionale sur une période de deux mois.

\section{Méthodologie : le modèle numérique et les forçages}

\subsection{SYMPHONIE, un modèle de courantologie 3D}

SYMPHONIE est un modèle de circulation océanique basé sur les équations primitives. Il est développé par l'équipe SIROCCO (MARSALEIX et al., 2008). La discrétisation du domaine de calcul est basée sur une méthode en différences finies. La grille verticale utilise des coordonnées sigma généralisées afin de raffiner la résolution près du fond et à la surface. Cette grille verticale est associée à une grille horizontale curviligne offrant un raffinement de la résolution à la côte tout en gardant des temps de calcul raisonnables. La résolution de la surface libre est faite de manière explicite par une méthode de pas de temps séparés (barotrope/barocline) (MARSALEIX et al., 2008). Le calcul des conditions sur les frontières ouvertes a été particulièrement soigné (MARSALEIX et al., 2006, 2009a), de même que la discrétisation du gradient de pression (MARSALEIX et al., 2009b). La paramétrisation de la turbulence dans la colonne d'eau s'appuie sur BOUGEAULT \& LACARRERE (1989). A l'interface air/mer les flux de quantité de mouvement et de chaleur sont calculés à partir de formules bulk (ESTOURNEL et al., 2009). Un point fort est la modélisation précise de la marée avec notamment la prise en compte des différents potentiels générateurs (PAIRAUD et al, 2008). L'implémentation dans SYMPHONIE des équations simplifiées de BENNIS et al. (2011) reposant sur la théorie glm2z-RANS de ARDHUIN et al. (2008) permet de modéliser les courants 3D induits par les vagues du plateau continental au début de la zone de surf (MICHAUD et al., 2012). Les paramètres liées aux vagues nécessaires au calcul dans SYMPHONIE sont donnés par le modèle de vagues WAVEWATCH III (TOLMAN, 2008, 2009 ; ARDHUIN et al., 2010). SYMPHONIE présente donc l'originalité d'embarquer les développements les plus récents en matière de couplage 3D vent/ vague.

\subsection{Les forçages}

Notre grille de calcul couvre environ 600 km autour de l'île de Taïwan et s'étend du Nord-Ouest du Pacifique jusqu'aux côtes Chinoises (figure 2A). Elle permet de modéliser l'impact des typhons provenant majoritairement du Sud/Sud-Est jusque dans le détroit de Taïwan. Elle a été construite à partir de la donnée bathymétrique GEBCO, 


\section{Thème 1 - Hydrodynamique côtière}

d'une bathymétrie issue de LALLEMAND S. et al. (1997) à l'occasion de ce travail et de la bathymétrie haute résolution réalisée sur la barrière sableuse de Wan-Tzu-Liao. La grille de calcul curviligne est de forme polaire centrée sur Taïwan, composé de 264684 points horizontaux et de 40 niveaux verticaux. Les calculs ont été effectués sur un cluster type HPC.

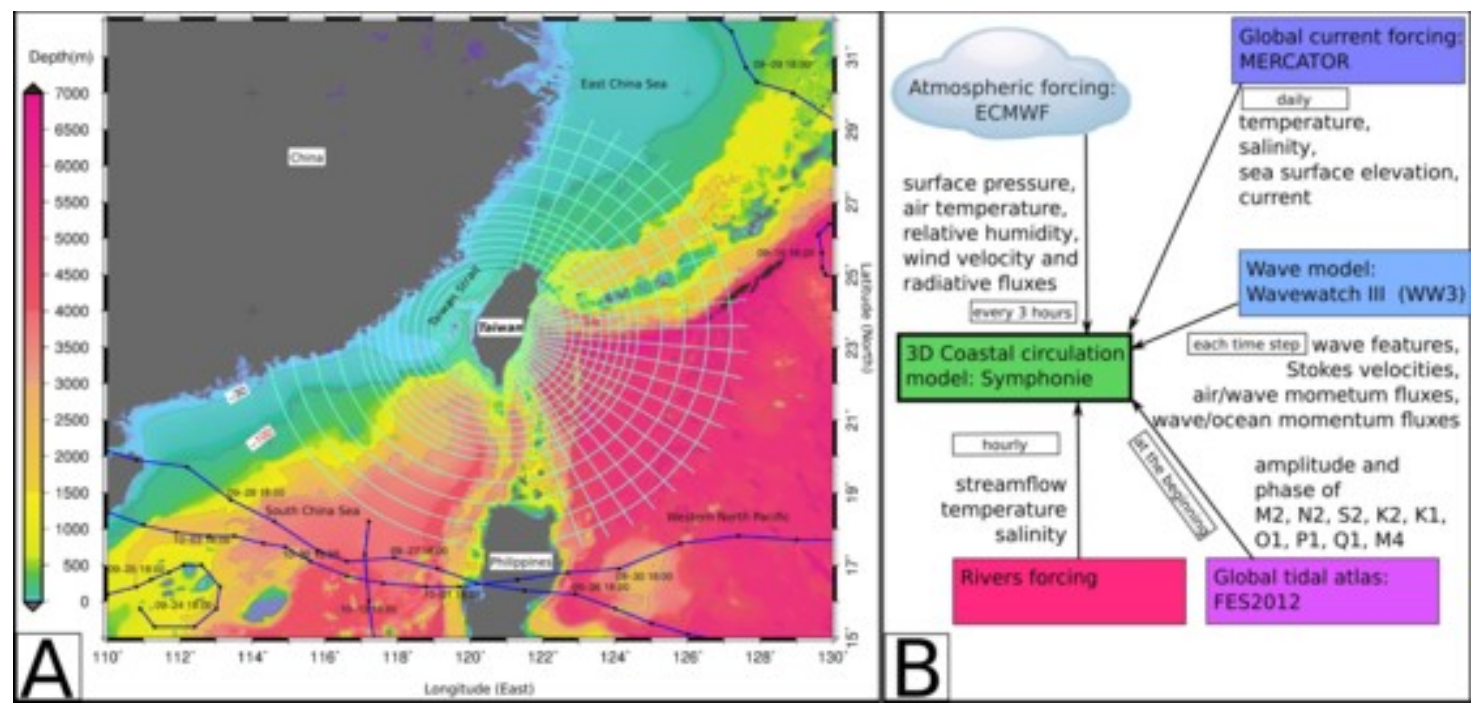

Figure 2. A) La grille de calcul polaire régionale avec une résolution de 460 mètres à la côte et de 5,60 kilomètres au large et le tracé des typhons pendant la période simulée. B) Aperçu des différents forçages pris en compte par SYMPHONIE.

Pour permettre des simulations réalistes, le modèle SYMPHONIE intègre convenablement la totalité des forçages météo-marins ayant un rôle sur la circulation et le niveau d'eau (figure 2B). Les variations du niveau d'eau et des courants sur les frontières latérales sont générées à partir des 9 principales composantes de la marée diurne et semi-diurne et à partir de la circulation océanique grande-échelle fourni par le système MERCATOR (MADEC, 2008). Une réanalyse harmonique sur notre domaine a été réalisée préalablement par SYMPHONIE sur 15 jours (composante par composante), à partir de l'atlas de marée globale FES2012 (LYARD et al., 2006). Les conditions atmosphériques sont fournies toutes les 3 heures par les données ECMWF (Era-Interim data ; DEE et al. (2011)) à une résolution horizontale de 0,25 degrés. La période simulée est Septembre 2011 à Octobre 2011 car il s'agit du début de la période de mesures couvertes par nos instruments.

\section{Les résultats}

Nous avons comparé les forçages météo de ECMWF avec les données de la bouée de CIGU (figure 3B). Durant cette période, les vents proviennent majoritairement du Nord avec une moyenne de 4,94 m/s. La vitesse du vent atteint un pic maximal à 13,8 m/s le 


\section{XIII ${ }^{\text {èmes }}$ Journées Nationales Génie Côtier - Génie Civil \\ Dunkerque, 2-4 juillet 2014}

3/10/2011. On observe que sur notre zone d'étude, les vents sont assez bien reproduits par ECMWF avec cependant une sous-estimation de leurs vitesses (figure 3B). Concernant les forçages hydro, les courants de surface ont une vitesse moyenne de 0,19 $\mathrm{m} / \mathrm{s}$ et les courants de fond de $0,16 \mathrm{~m} / \mathrm{s}$; ils proviennent majoritairement du Nord. L'élévation moyenne à CIGU est de $65 \mathrm{~cm}$.

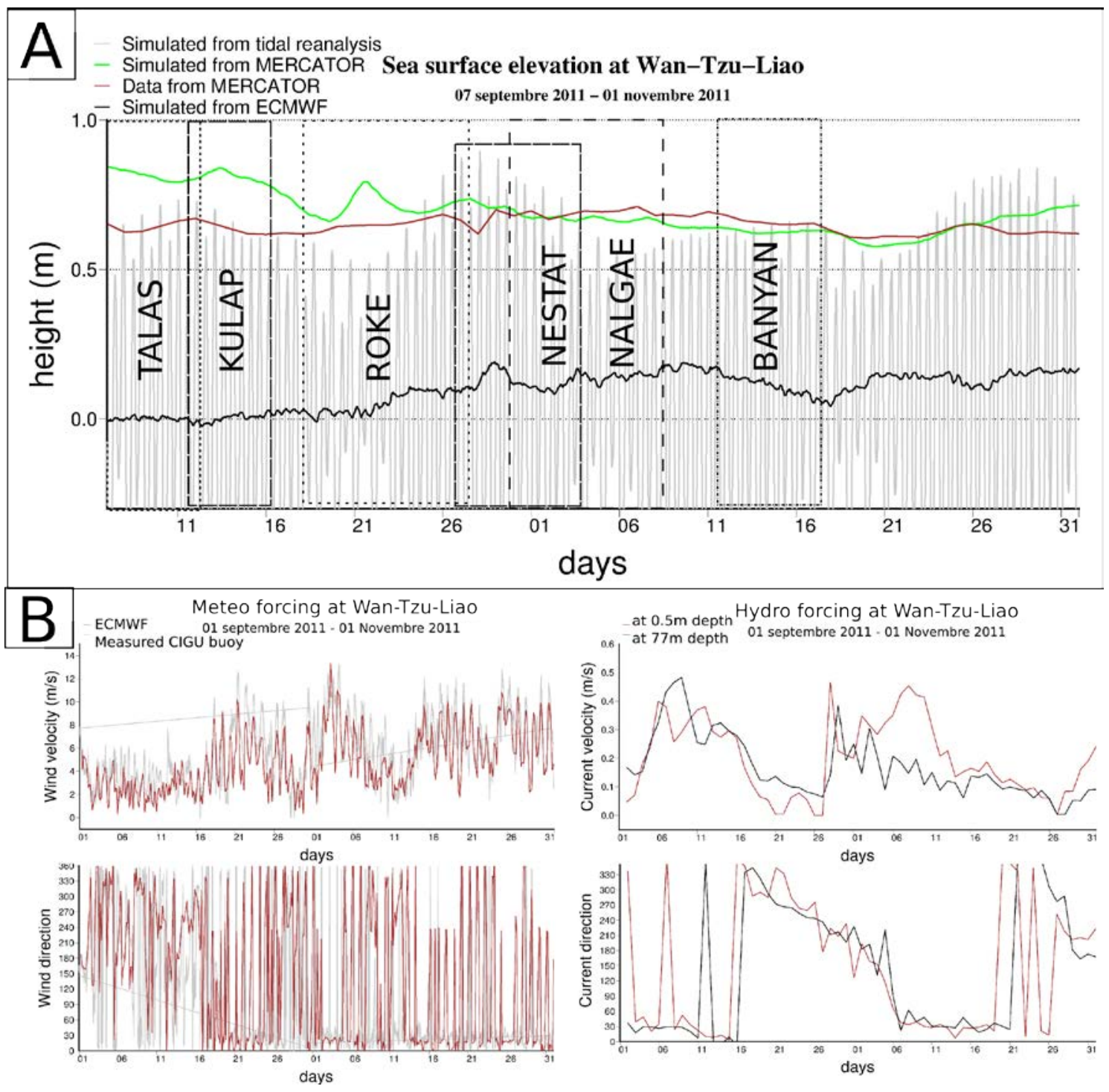

Figure 3. A) Analyse de l'élévation simulée de la surface selon les contributions (marée, courants globaux et des conditions atmosphériques) et les typhons à la bouée de CIGU entre le 7/09/2011 et 01/11/2011 ; B) Analyse des forçages météo et hydro à la bouée de CIGU entre le 01/09/2011 et 01/11/2011.

La figure 3A illustre le niveau d'eau simulé à la bouée de CIGU selon le forçage des marées, les courants globaux et les conditions atmosphériques. Le forçage par la marée reproduit bien le caractère semi-diurne à inégalités diurne (SIMON B. 2007) de la 
marée à Taïwan. L'élévation maximale due à la marée est de $77 \mathrm{~cm}$ le 28/09/2011. Le forçage par les courants globaux montre une élévation moyenne de $70 \mathrm{~cm}$ et une élévation maximale de $84 \mathrm{~cm}$ le 07/09/2011. Le forçage par les conditions atmosphériques montre une élévation maximale de $19 \mathrm{~cm}$ le 28/09/2011 et une élévation moyenne de $10 \mathrm{~cm}$.

Nous avons ensuite comparé la simulation couplant ces 3 forçages avec le marégraphe du port de Chung-Chin (figure 4). L'élévation moyenne est de $64 \mathrm{~cm}$ et le pic maximal est de 1,42 m le 29/10/2011. On peut noter que le modèle sous-estime l'élévation durant les plus hautes mers.

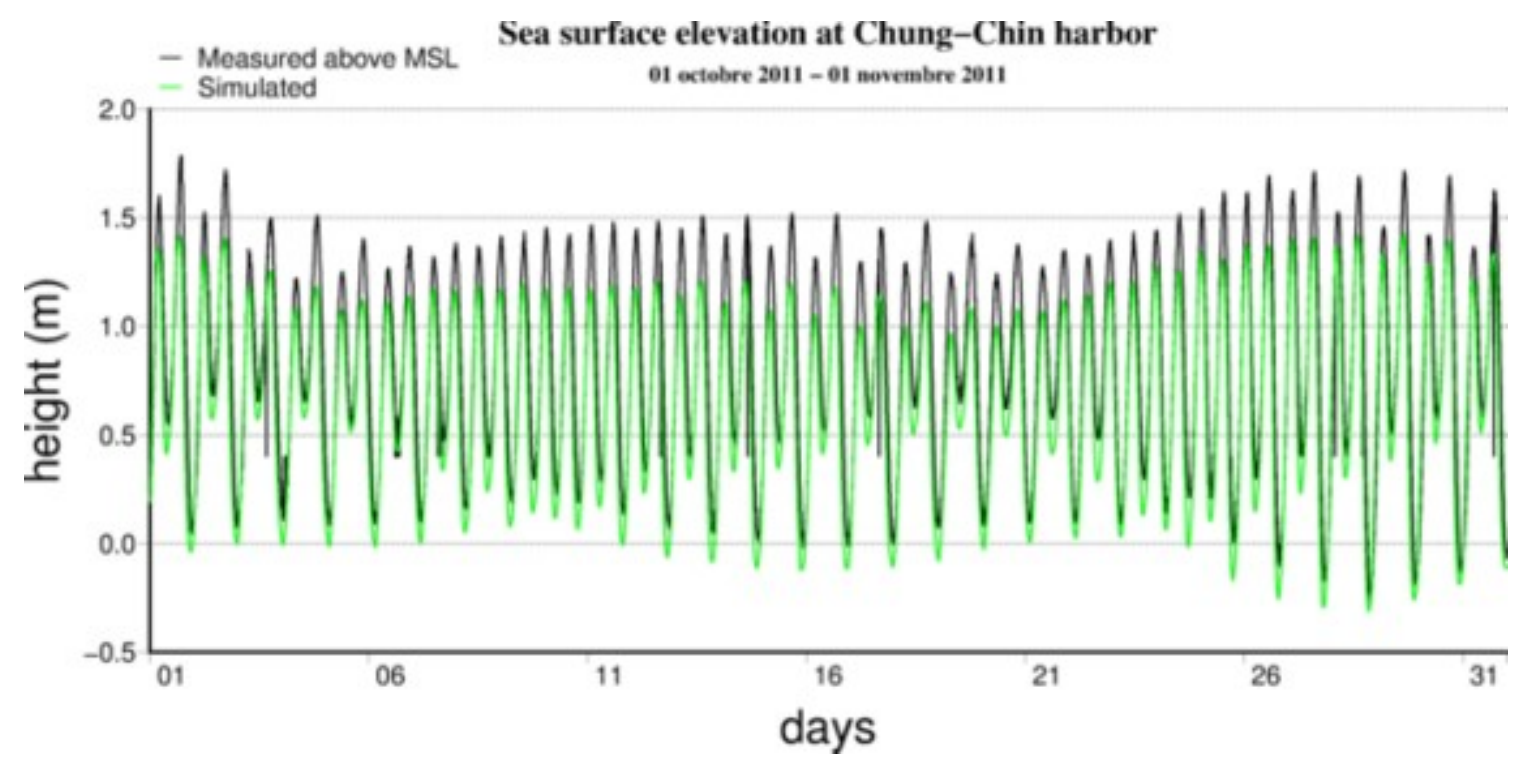

Figure 4. Comparaison du niveau d'eau simulé prenant compte de la marée, des conditions atmosphériques et de l'hydrodynamique grande échelle avec le marégraphe de Chung-Chin entre le 1/10/2011 et 01/11/2011.

\section{Discussion}

Lorsque l'on analyse les contributions séparément, on note que la marée et la circulation grande échelle jouent un rôle important sur l'élévation du niveau d'eau à la côte.

L'élévation induite par les courants semble plus importante lorsqu'ils proviennent de l'Ouest. Durant cette période où les typhons sont éloignés de notre zone d'étude, on s'aperçoit que la contribution des conditions atmosphériques est moindre. A ce stade du travail, nous pouvons supposer que la sous-estimation du modèle (de l'ordre de $9 \mathrm{~cm}$ sur les niveaux moyennés) vient de l'absence du forçage des vagues. L'intégration de ce forçage par le modèle Wave Watch III est en cours de réalisation ainsi que le forçage des rivières. Ces premiers résultats permettent d'analyser l'hydrodynamique local soumise à des conditions normales puisque les typhons sont éloignés de notre zone 


\section{XIII ${ }^{\text {èmes }}$ Journées Nationales Génie Côtier - Génie Civil \\ Dunkerque, 2-4 juillet 2014}

d'étude. La prochaine étape consiste à utiliser la même méthodologie en simulant une période soumise à des conditions extrême pour pouvoir comparer l'impact des typhons.

\section{Références bibliographiques}

ARDHUIN F., RASCLE N., BELIBASSAKIS K. (2008). Explicit wave-averaged primitive equations using a generalized lagrangian mean. Ocean Modelling, Vol. 20, pp 35-60. http://dx.doi.org/10.1016/j.ocemod.2007.07.001

ARDHUIN F., ROGERS E., BABANIN A. V., FILIPOT J. F., MAGNE R., ROLAND A., VAN DER WESTHUYSEN A., QUEFFEULOU P., LEFEVRE J. M., AOUF L., COLLARD F. (2010). Semiempirical dissipation source functions for ocean waves. Part I : Definition, calibration, and validation. Journal of Physical Oceanography, Vol. 40, pp 1917-1941. http://dx.doi.org/10.1175/2010JPO4324.1

BENNIS A., ARDHUIN F., DUMAS F. (2011). On the coupling of wave and threedimensional circulation models : Choice of theoretical framework, pratical implementation and adiabatic tests. Ocean Modelling, Vol. 40, pp 260-272. http://dx.doi.org/10.1016/j.ocemod.2011.09.003

BOUGEAULT P., LACARRERE P. (1989) Parametrization of orography-induced turbulence in a mesoscale model. Mon. Weather Rev. Vol. 117, pp 1872-1890. http://dx.doi.org/10.1175/1520-0493(1989)117<1872:POOITI>2.0.CO;2

CAMPMAS L., SABATIER F., MEULE S., LIOU J.-Y., PETITJEAN L., BOUTIN F., LEROUX-MALLOUF R., SOUS D., BOUCHETTE F. (2014) Multi-scale morphodynamics of sand barrier driven by monsoon/typhoon conditions. XIII ${ }^{\text {èmes }}$ Journées Nationales Génie Côtier - Génie Civil, Dunkerque. http://dx.doi.org/10.5150/jngcgc.2014.030

CHIOU M.-D., CHIEN H., CENTURIONI L.R., KAO C.-C. (2010). On the simulation of shallow water tides in the vicinity of the taiwan banks. Terr. Atmos. Ocean. Sci., Vol. 21(1), pp 45-69. http://dx.doi.org/10.3319/TAO.2009.03.18.01(IWNOP)

DEE D. et al. (2011). The ERA-Interim reanalysis : configuration and performance of the data assimilation system. Quarterly Journal of the Royal Meteorological Society, Vol. 137, pp 553-597. http://dx.doi.org/10.1002/qj.828

ESTOURNEL C., AUCLAIR F., LUX M., NGUYEN C., MARSALEIX P. (2009). "scale oriented" embedded modeling of the north-western mediterranean in the frame of MFSTEP. Ocean Science, Vol. 5, pp 73-90. http://dx.doi.org/10.5194/os-5-73-2009

LALLEMAND S. et al. (1997) Swath Bathymetry Reveals Active Arc-Continent Collision Near Taiwan, American Geophysical Union, Vol. 78, No 17, 173-175

LIN G.-F., HUANG P.-Y., CHEN G.-R. (2009). Using typhoon characteristics to improve the long lead-time flood forecasting of a small watershed. Journal of Hydrology, Vol. 380, pp 450-459. http://dx.doi.org/10.1016/j.jhydrol.2009.11.019 
LYARD F., LEFEVRE F., LETELLIER T., FRANCIS O. (2006). Modelling the global ocean tides : modern insights from FES2004. Ocean Dynamics, Vol. 56, pp 394-415. http://dx.doi.org/10.1007/s10236-006-0086-x

MADEC G. (2008). NEMO ocean engine. Note du Pole de modélisation, Institut PierreSimon Laplace (IPSL), France. No 27.

MARSALEIX P., AUCLAIR F., ESTOURNEL C. (2006). Considerations on open boundary conditions for regional and coastal ocean models. Journal of Atmospheric and Oceanic Technology, Vol. 23, pp 1604-1613. http://dx.doi.org/10.1175/JTECH1930.1

MARSALEIX P., AUCLAIR F., FLOOR J. W., HERRMANN M. J., ESTOURNEL C., PAIRAUD I., ULSES C. (2008). Energy conservation issues in sigma-coordinate freesurface ocean models. Ocean Modelling, Vol. 20, pp 61-89. http://dx.doi.org/10.1016/j.ocemod.2007.07.005

MARSALEIX P., AUCLAIR F., ESTOURNEL C. (2009b). Low-order pressure gradient schemes in sigma coordinate models : The seamount test revisited. Ocean Modelling, Vol. 30, pp 169-177. http://dx.doi.org/10.1016/j.ocemod.2009.06.011

MICHAUD H., MARSALEIX P., LEREDDE Y., ESTOURNEL C., BOURRIN F., LYARD F., MAYET C., ARDHUIN F. (2012). Three-dimensional modelling of waveinduced current from the surf zone to the inner shelf. Ocean Science, Vol. 8, pp 657-681. http://dx.doi.org/10.5194/os-8-657-2012

PAIRAUD I. L., LYARD F., AUCLAIR F., LETELLIER T., MARSALEIX P. (2008). Dynamics of the semi-diurnal and quarter-diurnal internal tides in the Bay of Biscay. Part 1: Barotropic tides, Continental Shelf Research, Vol. 28, pp 1294-1315. http://dx.doi.org/10.1016/j.csr.2008.03.004

SIMON B. (2007). La marée : La marée océanique côtière. Institut océanographique éditeur.

TOLMAN H. (2008). A mosaic approach to wind wave modeling. Ocean Modelling, Vol. 25, pp 35-47. http://dx.doi.org/10.1016/j.ocemod.2008.06.005

TOLMAN H. (2009). User manual and system documentation of WAVEWATCH-III version 3.14. Technical report, NOAA/NWS/NCEP/M-MAB. 